\title{
Effectiveness of neurorehabilitation treatment for individuals with brain injury or stroke
}

Treatment efficacy concerns the evaluation of therapeutic interventions that are studied under controlled conditions. Treatment effectiveness involves the assessment of therapeutic interventions that work in the real world. Although a number of published articles and meta-analyses have dealt with the issue of efficacy, few have addressed the issue of effectiveness. Perhaps the main reason for the dearth of articles on effectiveness is that neurorehabilitation may take several years or a lifetime to achieve. It may therefore be difficult to follow a patient's recovery across such a lengthy timespan or to measure improvement in ways that family members and survivors are likely to understand. The goal of this issue is to present real world applications of neurorehabilitation that showcase a diverse spectrum of effective therapeutic interventions after brain injury and stroke. For example, two articles illustrate successful applications of the Feuerstein Mediated Learning Experience and Instrumental Enrichment model which has been used primarily in the European Union and Israel for treating brain injury and stroke. Two other articles evaluate the effectiveness of Constraint Induced Aphasia Therapy and group therapy techniques for treating Aphasia. These articles illustrate the potential and limitations when using group therapy for brain injury rehabilitation. Another article deals with transcortical stimulation as an adjunct therapy for treating Aphasia.

Two additional articles concern treatment of academic difficulties. One study illustrates the successful treatment of a patient with Balint's Syndrome which is a rare condition, often associated with brain damage secondary to hypoxic. The authors detail an especially clever application of errorless learning that enabled the patient to re-learn letters of the alphabet and to read words. A second article is a systematic review of pediatric cognitive rehabilitation which focuses on the application of these techniques for improving executive difficulties with students in the public elementary and middle school systems.

There are a variety of other articles in this issue that concern lesser known methods of effective treatment. For example, emotional and executive difficulties are often reported after brain injury and stroke. Part of the problem of dealing with these issues is that there are few techniques for accurately measuring these deficits or for treating them. The measurement issue is addressed in four articles that explore the notion of self-appraisal, reflective appraisal, and the Dysexecutive Syndrome after ABI. Two of these studies show how the patients' self-perception can be assess within the context of a standard psychological evaluation process. A third study demonstrates the effectiveness of a comprehensive holistic neuropsychological rehabilitation program for reducing reported symptoms of dysexecutive behavior and career strain following ABI. Survivors often experience emotional volatility and anger after ABI which is the subject of a fourth article that showcases the effectiveness of perspective training for treating anger related issues after $\mathrm{ABI}$.

This issue also addresses several other topics that are important for therapists, survivors, and their families. Brain injury often results in Apraxia which is addressed in an article that reviews the evidence 
base on effectiveness of compensatory approaches for treating apraxia. Another article surveys the existing literature on Animal Assisted Therapy and suggests ways in which it can be used as an adjunct to effective therapy during the recovery process. Survivors' emotional volatility may also lead to legal problems which was the impetus for another study of jurors perception of defendants with and without ABI. This article demonstrates how providing a juror with information about the consequences of brain injury is sufficient to change their sentencing of defendants with ABI.

The characteristics of these therapies that makes them effective is that they are adaptable, measurable, and they provide gains that are tangible for survivors and family members. Most involve interventions that can be modified to fit the patient's needs. Success can also be measured unambiguously in terms of task performance and ability to function in everyday life rather than self-report. Many can be carried on by family members after formal therapy sessions are ended which is essential for sustaining gains. All of these therapies are evidence based which makes them excellent candidates for future research and development.

Guest Editor

Rick Parente

Department of Psychology

Towson University

8000 York Road

Timonium, MD 21252, USA

E-mail: fparente@towson.edu 